\title{
Investigating the peak energy-intensity correlation for GRB Physics and Cosmology
}

\author{
Disha Sawant ${ }^{* 1,2,3,4}$ \\ ${ }^{1}$ University of Ferrara, Via Saragat-1, Block C, Ferrara 44122, Italy \\ ${ }^{2}$ University of Nice, 28 Avenue Valrose, Nice 06103, France \\ ${ }^{3}$ IRAP Erasmus PhD Program, European Union \\ ${ }^{4}$ INAF - IASF Bologna, Via P. Gobetti 101, Bologna 41125, Italy \\ E-mail: sawantefe.infn.it

\section{Lorenzo Amati ${ }^{4,5}$} \\ ${ }^{4}$ INAF - IASF Bologna, Via P. Gobetti-101, Bologna 41125, Italy \\ ${ }^{5}$ ICRANet, Piazzale Aldo Moro-5, Rome 00185, Italy \\ E-mail: amati@iasfbo.inaf.it
}

\begin{abstract}
Gamma Ray Bursts (GRBs) radiate up to $10^{54}$ erg of energy isotropically $\left(\mathrm{E}_{\text {iso }}\right)$ and they can be observed in a wide range of redshift (from $\sim 0.01$ up to $\sim 9$ ). Such enormous energetics and high redshift make these phenomena very promising to shed light on the history and evolution of the universe. The major problem in utilizing the GRBs as cosmological tools is to find a way to standardize them, in a way similar to, e.g. SNe Ia. In this respect, the correlation between spectral peak energy $\left(\mathrm{E}_{p, i}\right)$ and the "intensity" is the most favorable and investigated criterion. Indeed, it has been shown that, through the $\mathrm{E}_{p, i}-\mathrm{E}_{i s o}$ correlation, the present data set of GRBs can already provide independent evidence of $\Omega_{M} \sim 0.3$ for a flat Universe. Here we investigate and compare the correlation of $\mathrm{E}_{p, i}$ with different intensity indicators (e.g., radiated energy, average and peak luminosity, bolometric vs. monochromatic quantities, etc.) both in terms of intrinsic dispersion of and accuracy for estimating $\Omega_{M}$. The results of the comparisons lead us to verify the reliability of the correlations for both GRB physics and their standardization for cosmology.
\end{abstract}

Swift: 10 Years of Discovery,

2-5 December 2014

La Sapienza University, Rome, Italy

*Speaker. 


\section{Introduction}

The typical spectra of GRBs prompt emission can be described by the Band function (Band et al. 1993)[1], which is a smoothly broken power law with the spectral parameters namely the low energy index $\alpha$, the high energy index $\beta$, the break energy $\mathrm{E}_{0}$ and the overall normalization. In this model, if $\beta<-2$ then the $v \mathrm{~F}_{v}$ (in units of Energy) spectral peak energy is given by $\mathrm{E}_{p}=\mathrm{E}_{0} \cdot(2+\alpha)$, where $\mathrm{E}_{p}$ is the photon energy at which the energy spectrum reaches the maximum. For those GRBs with reliable estimates of the redshift and a good spectral characterization, it is then possible to derive the cosmological rest-frame peak energy $\mathrm{E}_{p, i}$. The correlation of $\mathrm{E}_{p, i}$ with the isotropic-equivalent radiated energy $\left(\mathrm{E}_{i s o}\right)$ or peak luminosity $\left(\mathrm{L}_{p, i s o}\right)$ is the most investigated tool for standardizing long GRBs and exploiting the combination of their huge luminosities (more than $10^{52} \mathrm{erg} / \mathrm{s}$ ) and redshift distribution extending up to 9 (i.e. much beyond that of $\mathrm{SNe}$ Ia) for measuring cosmological parameters and, in perspective, the properties of dark energy.

The main issue regarding this standardization has always been the observed scatter. In addition, in the past years there have been discussions about the validity and reliability of this correlation. Under these respects, a vital step is to consider the correlation of $\mathrm{E}_{p, i}$ with all the possible intensity indicators related to GRBs. This comparison between purely observed quantities can interpret the possible causes of the dispersion and hence can point out the best candidate for standardizing GRBs with least possible scatter. This comparison is essential also for the better understanding of the selection and instrumentation biases affecting the standardization of GRBs.

In this short paper, we report partial and preliminary results of a systematic data collection and analysis aimed at comparing the different $\mathrm{E}_{p, i}$ - "Intensity" correlations both from the point of view of their dispersion and their accuracy in the estimate of cosmological parameters and their relevance for understanding GRB physics and sub-classes.

\section{Data Sample}

We collect the spectral information of GRBs with measured redshift from February 1997 to September 2013. Our database includes redshift (z), both energy indices ( $\alpha$ and $\beta$ ), the peak energy $E_{p}$ computed from the break energy $E_{0}, t_{90}$, exposure time, the fluence and the value of peak flux. The redshift distribution covers a broad range $(0.033 \leq z<9.0)$ thus extending far beyond that of Type Ia SNe $(z \leq 1.7)$. For the oldest GRBs (BeppoSAX, BATSE, HETE-2) and other GRBs up to mid 2008, the data was adapted from Amati et al. 2008[2].

The criteria behind selecting the measurements from a particular mission are based on following conditions:

1. The observations were preferred for which the exposure time was at least $2 / 3$ rd of the whole event duration.

2. Given the broad energy band and good calibration, Konus- WIND and Fermi/GBM were chosen whenever available. For Konus- WIND, the measurements were taken 
from the official catalog (Ulanov et al. 2005[3]) and from GCN archives (http: //gcn.gsfc.nasa.gov/gcn3_circulars.html). In case of Fermi/GBM, the observations were derived from Gruber at al. 2012[4] as the official literature and from several other papers (e.g., Ghirlanda et al. 2004[5], Ghirlanda et al. 2005[6], Friedman- Bloom 2005[7], etc.). The observations from SUZAKU were not considered as the uncertainties in the calibration are higher and also due to the fact that it works in a narrow energy band.

3. The SWIFT BAT observations were chosen when no other preferred missions (KonusWIND , Fermi/GBM) were able to provide information. Also, it was considered only for the GRBs when the value of $E_{p, o b s}$ was within the energy band of the instrument. For Swift GRBs, the $\mathrm{E}_{p, i}$ value derived from BAT spectral analysis alone were conservatively taken from the results reported by the BAT team (Sakamoto et al. 2008 $\mathrm{a}[8], \mathrm{b}[9])$. Other BAT $\mathrm{E}_{p, i}$ values reported in the literature were not considered, because either they were not confirmed by Sakamoto et al. $(2008 \mathrm{a}, \mathrm{b})$ refined analysis (e.g., Cabrera et al. 2007[10]) or they are based on speculative methods (Butler et al. 2007[11]). The GCN circulars were also used when needed.

When we came across more than one mission giving out good observations based on the criteria explained above, we took into account the values and uncertainties of all those observations (hence more than one set for some finely observed GRBs). When the observations were to be included in the data sample, we made sure that the uncertainty on any value doesn't go below $10 \%$ in order to account for the instrumental capabilities, etc. So, when the error was lower, we assumed it to be $10 \%$.

When available, the Band model (Band et al. 1993[1]) was considered since the Cut-off power law tends to overestimate the value of $\mathrm{E}_{p, i}$.

\section{Correlation of $\mathrm{E}_{p, i}$ with different "intensity" indicators}

The intensity indicators considered and computed in our analysis are:

- $\mathrm{E}_{\text {iso }}$ : The total radiated energy, computed by integrating the spectrum in a standard energy band and assuming isotropic emission.

- $\mathrm{L}_{\text {iso }_{T 90}}$ : The isotropic luminosity averaged over the T90 duration: The start of the T90 interval is defined by the time at which $5 \%$ of the total fluence has been detected and the end of the T90 interval is defined by the time at which $95 \%$ of the fluence been detected. In our case, the luminosity is integrated over the T90.

- $\mathrm{L}_{i s o_{E x p}}$ : The isotropic luminosity averaged over the exposure time: Exposure time is the interval (in seconds relative to trigger time) used in the spectral fits over the duration of the burst. So the luminosity is computed with respect to this exposure time.

- $\mathrm{L}_{p}$ : The luminosity computed at the peak of the spectra. 
All the quantities were computed over "Bolometric" (in the commonly used 1-10000 keV energy band in the cosmological rest frame) and also over "monochromatic" range (computed at at the peak $\left(\mathrm{E}_{p}\right)$ of the $v \mathrm{~F}_{v}$ spectrum.
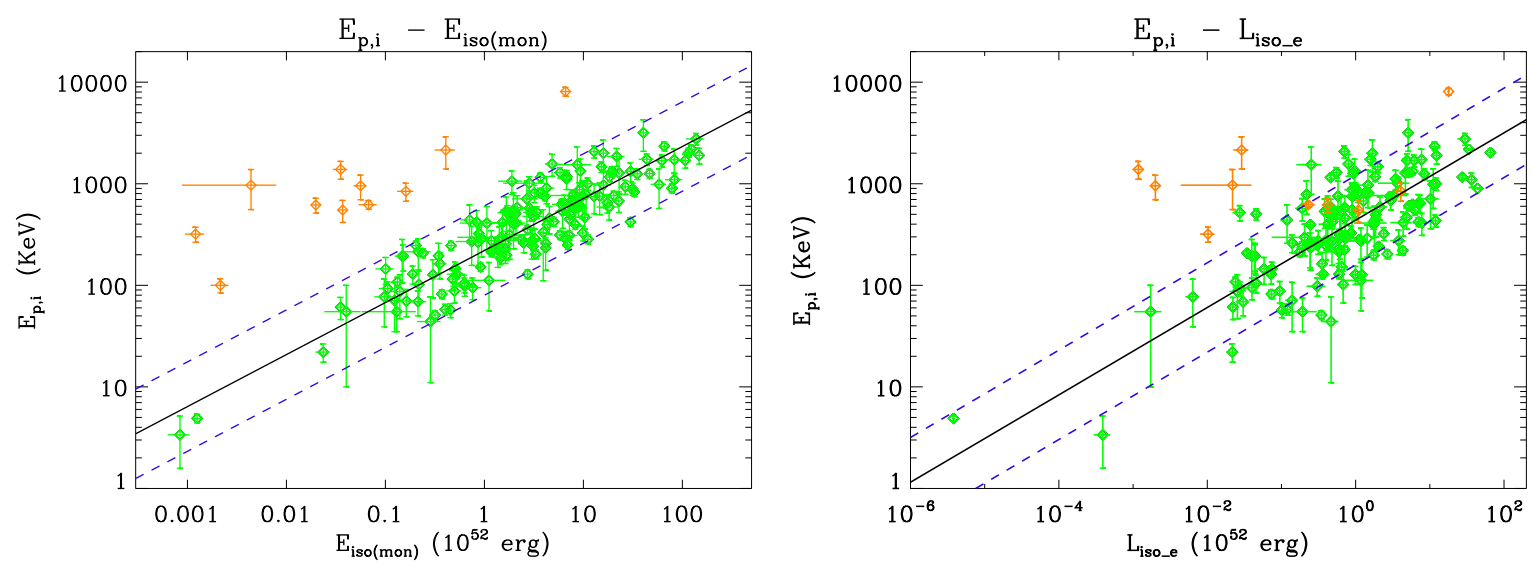

Figure 1: The left panel shows the monochromatic $\mathrm{E}_{p, i}-\mathrm{E}_{i s o}$ correlation where we have only considered the $\mathrm{E}_{\text {iso }}$ value at the peak of the energy spectrum. The right panel shows the $\mathrm{E}_{p, i}-\mathrm{L}_{i s o}$ correlation with respect to the exposure time. In both the plots, the plain black line depicts the best fit line for this correlation and the $2 \sigma$ blue dashed lines are the scatter limits of $\mathrm{E}_{p, i}-\mathrm{E}_{i s o}$ bolometric (Amati) correlation.

The fitting parameters (slope, normalization and dispersion) were estimated by adopting the statistical method proposed by Reichart et al. (2001)[12] which deals with fitting of the data points affected by extrinsic scatter in addition to the statistical uncertainties. Some of the results are graphically shown in Figure 1 and reported in Table 1. As a reference for comparing the scatter of the different correlations, we use the bolometric $\mathrm{E}_{p, i}$ $\mathrm{E}_{\text {iso }}$ (Amati) correlation.

From these results, we can draw the following preliminary considerations:

1. The $\mathrm{E}_{p, i}-\mathrm{E}_{i s o}$ correlation still remains the least scattered out of all the correlations considered. This can be explained mainly due to the fact that $\mathrm{E}_{\text {iso }}$ takes automatically into account the fact that the brightest parts of a GRB are those determining the $\mathrm{E}_{p}$ value of the time-averaged spectrum. This is not considered while using the average luminosity (either over exposure or T90) which is affected by the assumption that all the time bins of the GRB equally contribute to the average $\mathrm{E}_{p, i}$.

2. The correlation of $\mathrm{E}_{p, i}$ with monochromatic quantities is less scattered with respect to what found with the bolometric ones (in table and figures we show the $\mathrm{E}_{p, i}-\mathrm{E}_{\text {isomon }}$, the least scattered among the investigated correlations). This shows that a fraction of the dispersion is introduced in the extrapolation to a bolometric energy band due to the uncertainties on the fitting parameters $\alpha$ and $\beta$.

3. While considering the average luminosity instead of total radiated energy, the merging of some of the short GRBs into the long GRBs' populated region is observed. 
This may be pointing at the necessity of better understanding of classification of GRBs and of the their physical origin.

\section{Implications on the Cosmology}

We also investigated the accuracy of each considered correlation for cosmology, by following the maximum likelihood method that takes into account the uncertainties in both the $X$ and $Y$ quantities and the extra variance $\sigma_{\text {ext }}$ proposed by Reichart et al. (2001)[12] and the method established by Amati et al. (2008)[2]. Indeed, these authors found that, the $-\log$ (likelihood) of the $\mathrm{E}_{p, i}-\mathrm{E}_{i s o}$ as a function of the value of $\Omega_{M}$ assumed for the computation of $\mathrm{E}_{\text {iso }}$ within a flat $\Lambda \mathrm{CDM}$ scenario shows a nice parabolic shape, with a minimum at $\Omega_{M} \sim 0.30$.

We repeated the same analysis for all studied correlations. We observe that the dispersion of the correlations varies uniquely. Also, the minimization of $\Omega_{M}$ varies but still remains considerably near to 0.3 .

For all the correlations $\Omega_{M}$ shows a minimum around 0.3, although it is important to notice that for $\mathrm{E}_{p, i}-\mathrm{E}_{i s o}$ correlations for both bolometric and monochromatic computations, point out the minimization of $\Omega_{M}$ more accurately. The distinctive advantage of considering monochromatic frame is that the fit results are independent on $\alpha$ and $\beta$ and their uncertainties.

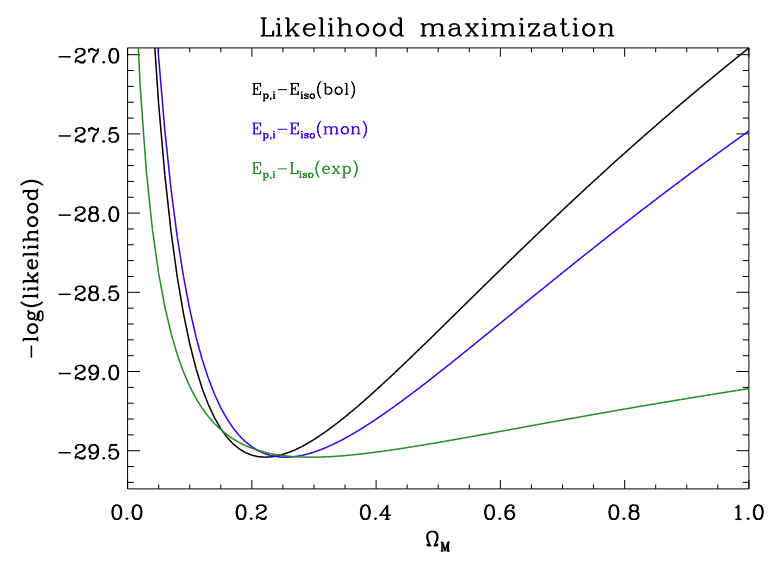

Figure 2: Likelihood maximization of some considered correlations as a function of $\Omega_{M}$ for flat CDM cosmology.

Table 1: Fit parameters of various GRB correlations

\begin{tabular}{lcccc}
\hline \hline Correlation & normalization & slope & $\sigma$ & $\Omega_{M}$ with 68\% C.L. \\
\hline \hline $\mathrm{E}_{p, i}-\mathrm{E}_{\text {iso }}$ bolometric & $1.95_{-0.03}^{+0.04}$ & $0.54_{-0.02}^{+0.03}$ & $0.22_{-0.02}^{+0.02}$ & $0.22_{-0.12}^{+0.25}$ \\
$\mathrm{E}_{p, i}-\mathrm{L}_{i s o}$ for exposure time & $2.64_{-0.03}^{+0.02}$ & $0.43_{-0.02}^{+0.03}$ & $0.30_{-0.02}^{+0.02}$ & 0.29 \\
\hline $\mathrm{E}_{p, i}-\mathrm{E}_{i s o}$ monochromatic & $2.34_{-0.02}^{+0.02}$ & $0.51_{-0.02}^{+0.02}$ & $0.19_{-0.02}^{+0.02}$ & $0.25_{-0.13}^{+0.18}$ \\
\hline
\end{tabular}




\section{Conclusions}

Our analysis shows that the $\mathrm{E}_{p, i}$-intensity correlation is robust, independent of the choice of luminosity indicator. $\mathrm{E}_{i s o}$ appears as the best intensity indicator, especially if considered for monochromatic range (less bias due to extrapolation).

Some of the short GRBs are found to be lying inside the region which is dominated by long GRBs when considered the luminosity instead of radiated energy. Hence they may be shedding some light on the ideas behind the physical origin and differentiation of SGRBs- LGRBs.

\section{References}

[1] D. Band, et al., BATSE observations of gamma-ray burst spectra. I-Spectral diversity, ApJ, 413, 281 (1993).

[2] L. Amati, C. Guidorzi, F. Frontera, et al., Measuring the cosmological parameters with the $E_{p, i}-E_{i s o}$ correlation of Gamma-Ray Bursts, MNRAS, arXiv: 0805.0377 (2008).

[3] M. V. Ulanov, S. V. Golenetskii, D. D. Frederiks, et al., Fast spectral variability of GRBs with known redshifts, NCCGSPC, 28, 351 (2005).

[4] D. Gruber, J. Greiner, A. von Kienlin, et al., Rest-frame properties of gamma-ray bursts observed by the Fermi Gamma-Ray Burst Monitor, AEA, 531A, 20 (2011).

[5] G. Ghirlanda, G. Ghisellini \& D. Lazzati, The collimation-corrected GRB energies correlate with the peak energy of their $v F_{v}$ spectrum, ApJ, 616, 331 (2004).

[6] G. Ghirlanda, G. Ghisellini and C. Firmani, Gamma Ray Bursts as cosmological tools, MNRAS, (2005) (astro-ph/0502186).

[7] A. S. Friedman \& J. S. Bloom, Toward a more standardized candle using Gamma ray burst energetics and spectra, ApJ, Vol. 627, Issue 1, (2005) pp. 1-25 (astro-ph/0408413).

[8] T. Sakamoto, et al., The First Swift BAT Gamma-Ray Burst Catalog, ApJ, 175, 179 (2008).

[9] T. Sakamoto, et al., Global Properties of X-Ray Flashes and X-Ray-Rich Gamma-Ray Bursts Observed by Swift, ApJ, 679, 570 (2008).

[10] J. I. Cabrera, et al., Spectral analysis of Swift long GRBs with known redshift, MNRAS, (2007) (astro-ph/0704.0791).

[11] N. R. Butler, A Complete Catalog of Swift GRB Spectra and Durations: Demise of a Physical Origin for Pre-Swift High-Energy Correlations, ApJ, 656, 1001 (2007).

[12] D. E. Reichart, Dust Extinction Curves and LyÎs Forest Flux Deficits for Use in Modeling Gamma-Ray Burst Afterglows and All Other Extragalactic Point Sources, ApJ, 553, 235 (2001) (RM). 